\section{Bei multiresistenter Tuberkulose alle Chancen nutzen}

Olaru ID et al. Bedaquiline-based treatment regimen for multidrug-resistant tuberculosis. Eur Respir ] 2017; 49: pii: 1700742.doi:10.1183/ 13993003.00742-2017

Die Therapie einer multiresistenten Tuberkulose (MDR-TB) ist häufig nicht erfolgreich. Das kann an fehlenden effektiven Antibiotika liegen oder an einer extrem arzneimittelresistenten Tuberkulose (XDR-Tb). Bedaquilin ist ein neues Antibiotikum zur Kombination mit anderen TB-Medikamenten in dieser Situation. Aufgrund einer erhöhten Mortalität in klinischen Studien und der Gefahr einer QTc-ZeitVerlängerung wird es aber nur zurückhaltend eingesetzt.

Um die Datenlage außerhalb klinischer Studien zu verbessern, werteten loana D. Olaru vom Deutschen Zentrum für Infektionsforschung in Borstel und Kollegen retrospektiv die Daten von 30 Patienten mit MDR/CDR-TB aus, die zwischen 2011 und 2016 am nationalen Referenzzentrum für MDR-TB in Borstel mit Bedaquilin behandelt worden waren. Die median 29,5 Jahre alten Patienten stammten zu 90\% nicht aus Deutschland, 21 (70\%) kamen aus der früheren Sowjetunion. Alle Patienten hatten nach der Sputumkultur eine MDR-TB, definiert als eine Resistenz gegen Isoniazid und Rifampicin. Bei 6 (20\%) beschränkte sich die Resistenz darauf, bei 2 Patienten (7\%) wurde eine zusätzliche Resistenz gegen injizierbare Zweitlinien-Tuberkulostatika (Amikacin, Kanamycin oder Capreomycin), bei 7 (23\%) eine zusätzliche Fluorochinolonresistenz festgestellt. Eine XDR-TB, definiert als eine MDR-TB plus zusätzlich sowohl eine Resistenz auf Zweitlinien-Tuberkulostatika als auch Fluorochinolone, wiesen 15 Patienten (50\%) auf. 25 Patienten $(83 \%)$ waren gegen alle ErstlinienTherapeutika resistent.

\section{Ergebnisse}

Median wurden 6 wirksame Arzneimittel eingesetzt. Die Therapie mit Bedaquilin begann im Median 5,5 Tage nach Beginn der MDR-TB-Behandlung. Alle Patienten erhielten mindestens 6 Monate lang ein Bedaquilin-haltiges Regime.

War die Bedaquilintherapie im ersten Monat begonnen worden, lag die mediane Zeit bis zur Konversion im Ausstrich bei 52 Tagen (IQR 4-87 Tage), bis zur Konversion in der Sputumkultur bei 49
Tagen. Nach 6 Monaten zeigten alle Patienten in der Sputumkultur eine Konversion. Bei XDR-TB lag die mediane Zeit bis zur Konversion in Kultur mit 54 Tagen deutlich über der bei Patienten ohne XDR-TB (14 Tage).

Bedaquilin war gut verträglich und führte bei keinem Patienten zu einem Therapieabbruch. QT-Zeit-Verlängerungen traten bei 20 (95\%) von 21 Patienten auf, bei denen dies regelmäßig dokumentiert war. Es kam nicht zu kardialen Arrythmien und Todesfällen.

FAZIT

In einem Land mit geringer Tuberkuloseinzidenz und hohem Einkommensniveau (die Therapie kostet in Deutschland etwa 85000 Euro pro Patient) kann Bedaquilin hohe Sputumkultur-Konversionsraten bei MDR/XDR-TB erreichen. Trotz der QT-Zeit-Verlängerung bezeichnen die Autoren das Antibiotikum insgesamt als sicher und empfehlen den rationalen Einsatz bei allen Patienten mit MDR/XDR-TB, für die keine anderen Alternativen zur Verfügung stehen.

Friederike Klein, München 